INPLASY

PROTOCOL

To cite: Alshahrani et al. Effect of Lower Limb Constrained Induced Movement Therapy on Gait, Balance, and Cardiovascular parameters-A systematic review and Metaanalysis. Inplasy protocol 202170008. doi: 10.37766/inplasy2021.7.0008

Received: 03 July 2021

Published: 03 July 2021

Corresponding author: Kumar Gular

kmeny@kku.edu.sa

Author Affiliation: KING KHALID UNIVERSITY

Support: Self financed.

Review Stage at time of this submission: Data analysis.

Conflicts of interest: None declared.

\section{Effect of Lower Limb Constrained Induced Movement Therapy on Gait, Balance, and Cardiovascular parameters-A systematic review and Meta-analysis}

Alshahrani, MS1; Gular, K2; Tedla, JS3; Rengaramanujam, K4; Kakaraparthi, VN5; Dixit, S6; Reddy, RS7.

Review question / Objective: To find out the effects of lower limb Constrained Induced Movement Therapy on gait, balance, and cardiovascular parameters.

Condition being studied: Incidence of stroke more common in all age groups, both genders and, among low and middle socio-economic countries. The mortality and disability rate increased exceptionally in subacute and chronic strokes. The incidence, mortality and, disability rate of stroke stress the need for primary preventive measures and quality rehabilitation settings.

Information sources: Science Direct, Scopus, Web of Science, Google Scholar, PubMed, and PEDro Database were searched for relevant articles published in the English language between 2000 to 2020 .

INPLASY registration number: This protocol was registered with the International Platform of Registered Systematic Review and Meta-Analysis Protocols (INPLASY) on 03 July 2021 and was last updated on 03 July 2021 (registration number INPLASY202170008).

\section{INTRODUCTION}

Review question / Objective: To find out the effects of lower limb Constrained Induced Movement Therapy on gait, balance, and cardiovascular parameters.

Rationale: The significant improvements of the CIMT approach on upper extremity functions provoked the researchers to utilize it for lower extremity training among post-stroke subjects. The ability to walk depends on mobility, balance, and cardiorespiratory fitness determines community Participation,. Independent walking has a good association with gait functions, balance, and cardiovascular fitness; improvements in these functions 
will affect post-stroke subjects' walking ability.

Condition being studied: Incidence of stroke more common in all age groups, both genders and, among low and middle socio-economic countries. The mortality and disability rate increased exceptionally in subacute and chronic strokes. The incidence, mortality and, disability rate of stroke stress the need for primary preventive measures and quality rehabilitation settings.

\section{METHODS}

Search strategy: Science Direct, Scopus, Web of Science, Google Scholar, PubMed, and PEDro Database were searched for relevant articles published in the English language between 2000 to 2020. CIMT, mCIMT, forced use, stroke, subacute, acute, chronic stroke, CVA, gait parameters, balance, and lower limb functions are the keywords used to search appropriate studies.

Participant or population: First time or recurrent, hemorrhagic, or ischeamic post stroke subjects with age above 18 years were considered.

Intervention: Lower limb constrained induced movement therapy.

Comparator: Conventional treatment or any other neurorehabilitation techniques.

Study designs to be included: Randomized Controlled Trials.

Eligibility criteria: Randomized controlled trails (RCT's) which included first time or recurrent, hemorrhagic, or ischemic post stroke subjects with age above 18 years were considered. Studies which measured and analyzed the gait, balance, and cardiovascular parameters, were encompassed for the review.

Information sources: Science Direct, Scopus, Web of Science, Google Scholar, PubMed, and PEDro Database were searched for relevant articles published in the English language between 2000 to 2020.

Main outcome(s): Gait, balance, and cardiovascular outcomes Spatio-temporal, kinematic and kinetic outcomes will be considered. The scales that assessed static, dynamic and functional mobility components were considered. All vital signs along with scales assessing cardiovascular outcome will be included for review.

Additional outcome(s): Motor functions, Quality of Life (QOL), and Functional ambulation.

Data management: Patients' characteristics, Sample size, Eligibility criteria, Type and duration of constraint application, Outcome measurements, and 6. PEDro score used for data extraction. In case of discrepancy, the concerned author was contacted through email for further clarification or resolved through consensus.

Quality assessment / Risk of bias analysis: The methodological quality of studies intended to assess through PEDro scoring system Two independent reviewers will be involved in the assessment of the methodological quality of the studies. A third reviewer will be consulted and authorized to take decisions when a difference arose between the independent reviewers in assigning the scores for the studies. The RCTs were categorized as excellent, good, fair, and poor if they scored 9-10, 6-8, 4-5 and below 4 on PEDro scores respectively. The RCTs with PEDro $\geq 6$ is considered as Level 1 evidence and will be subcategorized based on the number of RCTs supporting the evidence statement into level $1 \mathrm{a}$ if 2 articles and level $1 \mathrm{~b}$ if one article supported respectively. Whereas the RCTs with level 2 evidence consist of a score less than 6 (PeDro <6).

Strategy of data synthesis: The amount of heterogeneity among the trails were assessed by 12 statistic. . We will consider an 12 value greater than $50 \%$ as substantial heterogeneity.fixed-effect or random- 
effects model were performed based on the results of 12 heterogeneity test and clinical heterogeneity. A pooled standardized mean difference was calculated for outcome measures which measures through different tools and polled mean difference will be calculated for outcome measures using single tool. We consider the clinical heterogeneity among studies, a random- effects model was applied with $95 \% \mathrm{Cl}$ using Review manager (non-Cochrane mode) for analysis.

Subgroup analysis: Subgroup analysis will be performed for chronicity, duration, and type of constrain.

Sensitivity analysis: Sensitivity will be assessed considering the following aspects: (a) random allocation, (b) concealed allocation, (c) methodological quality, (d) blinding of participants, (e) therapists blinding, (f) outcomes assessor blinding, (g) intention to treat analysis, and (h) dropouts.

\section{Language: English.}

\section{Country(ies) involved: Saudi Arabia.}

Other relevant information: A standardized mean difference will be used if the outcome measures were measured using different scales if not the treatment effects will be detailed through the mean difference in case of the same scale used for outcome measures at $95 \%$ confidence intervals $(95 \% \mathrm{Cl})$ for continuous data. If outcomes are dichotomous it will be reported through risk differences at $95 \% \mathrm{Cl}$.

Keywords: Stroke, Lower Limb CIMT, GAIT, Balance, Cardiovascular, systematic review.

Contributions of each author:

Author 1 - Mastour Saeed Alshahrani Framing objectives and methodology, reviewing the articles.

Email: msdalshahrani@kku.edu.sa

Author 2 - Kumar Gular - Performing quality assessment and statistical analysis of the included studies, drafting and approving the final manuscript.

Email: kmeny@kku.edu.sa

Author 3 - Jayashanker Tedla - Searching relevant articles and data extracting.

Email: jtedla@kku.edu.sa

Author 4 - Kangaraj Rengaramanujam Searching relevant articles and analyzing the results.

Email: krenga@kku.edu.sa

Author 5 - Venkata Nagaraj Kakaraparthi Statistical analysis of the included studies and writing discussion.

Email: vnraj@kku.edu.sa

Author 6 - Snehil Dixit - Performing risk of bias assessment and sensitivity.

Email: snehil@kku.edu.sa

Author 7 - Ravi Shankar Reddy Yerragondla - Analyzing the results and writing discussion.

Email: rshankar@kku.edu.sa 